\title{
Diversidade de Coleoptera (Arthropoda: Insecta) e atributos edáficos em sistemas de uso do solo no Planalto Catarinense
}

\author{
Coleoptera diversity (Artbropoda: Insecta) and soil properties under soil management systems in the highlands of Santa \\ Catarina state, Brazil
}

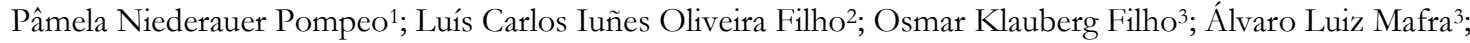 Carolina Riviera Duarte Maluche Baretta ${ }^{4}$; Dilmar Baretta ${ }^{5}$

\begin{abstract}
${ }^{1}$ Mestranda em Engenharia Florestal; Universidade do Estado de Santa Catarina (UDESC - CAV); pamniederauer@gmail.com 2Professor; Departamento de Agronomia; Universidade do Oeste de Santa Catarina (UNOESC); iunes1981@gmail.com 3Professor; Departamento de Solos e Recursos Naturais; UDESC-CAV; osmar.klauberg@,udesc.br, alvaro.mafra@udesc.br 4Professora, Programa de Pós-Graduação em Ciências Ambientais, UNOCHAPECÖ; carolmaluche@unochapeco.edu.br 5*Professor; Departamento de Zootecnia; UDESC Oeste; dilmar.baretta@udesc.br
\end{abstract}

Artigo enviado em 13/05/2016, aceito em 26/08/2016 e publicado em 13/10/2016.

\begin{abstract}
Resumo - Os coleópteros (Coleoptera) se distribuem em diferentes áreas e profundidades do solo e são importantes executores de diversos serviços ecossistêmicos. Seu estudo representa uma excelente ferramenta para elucidar os efeitos da perturbação antrópica sobre a biodiversidade e funções dos ecossistemas. O objetivo desse estudo foi avaliar a diversidade de famílias de coleópteros em diferentes sistemas de uso do solo (SUS), bem como a relação destes com os atributos edáficos. Os sistemas estudados foram: floresta nativa (FN), reflorestamento de eucalipto (RE), pastagem (PA), integração lavoura-pecuária (ILP) e lavoura com plantio direto (PD). As amostras foram coletadas em grade de amostragem de $3 \times 3$ pontos, distanciados entre si em $30 \mathrm{~m}$, nos períodos de inverno e verão, em três municípios do Planalto Sul Catarinense. Os coleópteros edáficos foram coletados pelos métodos de escavação "Tropical Soil Biology and Fertility - TSBF" e armadilhas "Piffall traps". Nos mesmos pontos coletaram-se amostras para determinação dos atributos físicos e químicos do solo. Utilizou-se análise estatística multivariada para a composição da comunidade, sendo, as variáveis ambientais consideradas como explicativas. Foram capturados 1.437 indivíduos, sendo as Famílias Staphylinidae, Carabidae e Scarabaeidae as mais representativas. Os sistemas RE e PD apresentaram maior diversidade de acordo com o índice de Shannon-Wiener $(\mathrm{H})$. As análises de componentes principais demonstraram distinção na distribuição dos invertebrados entre os diferentes SUS. As propriedades do solo contribuíram para explicar essa variação, tendo influência na diversidade de famílias de Coleoptera, destacandose os atributos matéria orgânica e porosidade que favoreceram a maior abundância de famílias em FN.
\end{abstract}

Palavras-chave - invertebrados, biodiversidade edáfica, ecologia de comunidades.

Abstract - Beetles (Coleoptera) are distributed in different areas and soil depths and they are important executors of several environmental services. Their study is an excellent tool to elucidate the effects of human disturbance on biodiversity and ecosystem functions. The aim of this study was to evaluate the diversity of Coleoptera families in different land use systems (LUS) and their relationship with soil properties. The systems were: native forest (NF), eucalyptus plantations (EP), pasture (PA), crop-livestock integration (CLI) and with no-tillage crop (NT). The samples were collected in sampling grid of $3 \times 3$ points apart from each other at $30 \mathrm{~m}$, in the periods of winter and summer, in three municipalities of the highlands of Santa Catarina state. Edaphic invertebrates were collected by methods of monoliths (Tropical Soil Biology and Fertility - TSBF) and Pitfall traps. In the same points samples soil was collected to determine the physical and chemical properties. The multivariate statistical analysis was used to the community composition, and the environmental variables were considered as explanations. 1.437 individuals were collected, the Staphylinidae, Carabidae and Scarabaeidae families was the most representative. The EP and NT systems showed the greatest diversity in accordance with the Shannon-Wiener index $(\mathrm{H})$. The principal component analysis showed distinction in the distribution of invertebrates between different LUS. Soil properties contributed to explain this variation, having influence on the diversity of Coleoptera families, highlighting the attributes organic matter and porosity that favored the greatest abundance of families in NF.

Keywords - invertebrates, edaphic biodiversity, community ecology. 


\section{INTRODUÇÃO}

A fauna, em conjunto com a microbiota e as raízes das plantas, compõe o sistema biológico do solo e atua em seus processos químicos, físicos e biológicos. Inclui muitos invertebrados com diferentes funções para a manutenção e sobrevivência de comunidades vegetais e animais. As alterações antrópicas provocadas no ambiente, podem influenciar esse sistema e seus organismos de forma direta ou indireta e em diversos níveis ou intensidades. Assim, a fragmentação dos habitats e a conversão de áreas com vegetação nativa em sistemas produtivos, podem modificar de forma drástica a biodiversidade no solo, decorrentes da exploração demasiada dos recursos naturais (BARETTTA et al., 2006). Não só os sistemas agropecuários modificam a dinâmica do solo, mas também os monocultivos florestais, pois reduzem a qualidade da serapilheira, levando a redução da biodiversidade de organismos edáficos (ASHFORD et al., 2013).

Os grupos edáficos se comportam de maneiras diferentes em seu habitat e respondem de forma imediata às alterações do ambiente, portanto, as alterações impostas as suas populações podem não ser uniforme em todos os táxons (LIIRI et al., 2012). Estudar essas modificações nas comunidades do solo pode ajudar a compreender e medir as consequências das perturbações impostas na paisagem. A entomofauna representa mais da metade dos animais existentes no planeta e sua utilidade como indicadores ecológicos é inquestionável, pois reagem rapidamente às mudanças, refletidas na sua densidade e diversidade, sendo geralmente de fácil coleta a campo e identificação (FAVERO; SOUZA; OLIVEIRA, 2011).

Dentre a ordem Coleoptera, existem famílias que são executoras de serviços do ecossistema, por exemplo Scarabaeidae com coleópteros que realizam escavação e posterior acúmulo e incorporação de matéria orgânica no solo em diferentes profundidades. Adicionalmente, existem famílias que atuam na decomposição de excretas e de resíduos de origem animal e vegetal, aeração do solo, transporte de matéria orgânica e no controle biológico (ALMEIDA; LOUZADA, 2009). Outro exemplo é a família Carabidae que fornece serviços importantes no controle de invertebrados considerados pragas e sementes de plantas daninhas (KROMP, 1999). Estes tem sido relacionados a qualidade do solo pois respondem as mudanças de temperatura, umidade, aplicação de herbicidas, adubação, alterações estruturais e por alguns grupos apresentarem larga distribuição e abundância, podem ser utilizados em avaliações de monitoramento contribuindo para verificar os efeitos das diferentes culturas sobre a biodiversidade (LEE; ALBAJES, 2016). Por estarem envolvidos nesses diversos processos edáficos alguns grupos de Coleoptera são associados a características físicas e químicas do solo como umidade, quantidade de nutrientes, além de serem afetados negativamente pela intensificação de uso e manejo dos sistemas de produção (FARIAS et al., 2015). Demonstrando assim que os coleópteros podem ser eficientes indicadores das condições ambientais.

Estudar a comunidade dos coleópteros representa um excelente foco para elucidar os efeitos da perturbação antrópica sobre a biodiversidade e funções do ecossistema, pois mesmo mudanças sutis, poderiam trazer consequências, em longo prazo, para a estrutura e diversidade das florestas e outros sistemas agrícolas (FAVERO; SOUZA; OLIVEIRA, 2011). A partir das considerações iniciais, as seguintes hipóteses foram testadas: i) os sistemas de uso e manejo do solo podem interferir na diversidade de famílias de Coleoptera; ii) as variáveis ambientais (tipo de vegetação, edafoclimáticas e atributos químicos e físicos do solo) podem contribuir para explicar a composição da comunidade e diversidade de famílias de coleópteros nos diferentes ambientes.

Diante do exposto, o objetivo do presente estudo foi avaliar a diversidade de famílias de Coleoptera em sistemas de uso do solo, bem como a sua relação com as variáveis ambientais (atributos físicos e químicos do solo) utilizadas como explicativas, visando compreender a distribuição destas no inverno e no verão do Planalto Sul Catarinense.

\section{MATERIAL E MÉTODOS}

\section{Locais de estudo}

O estudo foi conduzido nos municípios de Campo Belo do Sul, Lages e Otacílio Costa, no Planalto Sul Catarinense. Essa região caracteriza-se, de acordo com a classificação climática de Köppen, como subtropical úmido e possui um clima mesotérmico úmido (Cfb). Devido à sua formação geológica e a ação climática, há diversos tipos de solos na região, mas a maior parte caracteriza-se por ter profundidade média, com baixa a média fertilidade natural. Os solos estudados em Lages e Campo Belo do Sul são caracterizados como Nitossolo Bruno e em Otacílio Costa como Cambissolo Húmico.

Foram estudados cinco sistemas de uso do solo (SUS), envolvendo floresta nativa (FN), reflorestamento de eucalipto (RE), pastagem perene (PA), integração lavourapecuária (ILP) e plantio direto (PD), em cada município, totalizando 15 áreas de estudo, no Planalto Sul do Estado de Santa Catarina, Brasil, abrangendo os municípios de Campo Belo do Sul, Lages e Otacílio Costa (Tabela 1). Os 
três municípios da região foram selecionados de acordo com suas características geográficas, tipo de solo e histórico de manejo e considerados como as réplicas verdadeiras dos SUS.

As áreas de floresta nativa referem-se a remanescentes de Floresta Atlântica, com formação de Floresta Ombrófila Mista, consideradas como referências, por possuírem vegetação bem estabelecida, apresentando sub-bosque com trilhas e entrada ocasional de animais de produção.

Tabela 1 - Características dos sistemas Floresta nativa (FN), Reflorestamento de eucalipto (RE), Pastagem perene (PA), Integração lavoura-pecuária (ILP) e Plantio direto (PD), no Planalto Sul Catarinense.

\begin{tabular}{|c|c|c|c|c|}
\hline Município & Sistema & $\begin{array}{c}\text { Tamanho } \\
\text { (ha) }\end{array}$ & $\begin{array}{c}\text { Coordenadas } \\
\text { geográficas }\end{array}$ & $\begin{array}{l}\text { Altitude } \\
(\mathrm{m})\end{array}$ \\
\hline \multirow{5}{*}{ Lages } & $\mathrm{FN}$ & 100 ha & $\begin{array}{c}\text { S27 } 47.963 \\
\text { W50 } 35.743\end{array}$ & 895 \\
\hline & RE & $29 \mathrm{ha}$ & $\begin{array}{c}\text { S27 } 47.752 \\
\text { W50 } 36.069\end{array}$ & 852 \\
\hline & PA & 100 ha & $\begin{array}{c}\text { S27 } 47.873 \\
\text { W50 36.000 }\end{array}$ & 858 \\
\hline & ILP & 10 ha & $\begin{array}{c}\text { S27 } 47.544 \\
\text { W50 } 35.802\end{array}$ & 873 \\
\hline & PD & $7 \mathrm{ha}$ & $\begin{array}{c}\text { S27 } 47.123 \\
\text { W50 } 35.972 \\
\end{array}$ & 883 \\
\hline \multirow{5}{*}{$\begin{array}{l}\text { Campo } \\
\text { Belo do } \\
\text { Sul }\end{array}$} & $\mathrm{FN}$ & $5 \mathrm{ha}$ & $\begin{array}{c}\text { S27 52.943 } \\
\text { W50 } 39.338\end{array}$ & 1.016 \\
\hline & RE & $1,2 \mathrm{ha}$ & $\begin{array}{c}\text { S27 } 53.363 \\
\text { W50 } 39.056\end{array}$ & 989 \\
\hline & PA & 30 ha & $\begin{array}{c}\text { S27 } 52.130 \\
\text { W50 } 39.175\end{array}$ & 1.004 \\
\hline & ILP & 25 ha & $\begin{array}{c}\text { S27 } 52.131 \\
\text { W50 } 39.980\end{array}$ & 947 \\
\hline & PD & 55 ha & $\begin{array}{c}\text { S27 } 52.365 \\
\text { W50 40.366 }\end{array}$ & 923 \\
\hline \multirow{5}{*}{$\begin{array}{l}\text { Otacílio } \\
\text { Costa }\end{array}$} & $\mathrm{FN}$ & $3 \mathrm{ha}$ & $\begin{array}{c}\text { S27 35.674 } \\
\text { W49 50.927 }\end{array}$ & 919 \\
\hline & RE & 2,4 ha & $\begin{array}{c}\text { S27 } 33.446 \\
\text { W49 } 56.879\end{array}$ & 855 \\
\hline & PA & 10 ha & $\begin{array}{c}\text { S27 } 37.151 \\
\text { W49 } 51.461\end{array}$ & 900 \\
\hline & ILP & 22 ha & $\begin{array}{c}\text { S27 } 37.110 \\
\text { W49 } 51.418\end{array}$ & 902 \\
\hline & PD & 80 ha & $\begin{array}{c}\text { S27 } 29.063 \\
\text { W49 } 54.215\end{array}$ & 879 \\
\hline
\end{tabular}

Os plantios de eucalipto foram compostos por árvores de Eucalyptus dunni Maiden e Eucalyptus benthamii Maiden \& Cambage com 7, 20 e 21 anos, sendo áreas originalmente de campo nativo e usadas como pastagem, anteriormente à conversão para reflorestamento, com entrada de animais, exceto em Otacílio Costa, onde antes havia plantio de Pinus no local. Já as pastagens, foram áreas de campo nativo (vegetação nativa) e não apresentavam qualquer tipo de manejo, mas sofreram processo de queimadas, com densidade de 0,4 a 1,5 bovinos ha ${ }^{-1}$ e estavam nesse sistema entre 12 a 15 anos.

As áreas de integração lavoura-pecuária, apresentavam plantação sob semeadura direta, em Lages e Otacílio Costa foi plantado soja (Glycine max L.) no verão e azevém (Lolium sp.) e aveia (Avena sp.) no inverno. Já em Campo Belo do Sul, no verão, havia plantio de soja e no inverno era utilizada como pastagem ou trigo (Triticum sp.), lotação de 2 animais ha- ${ }^{-1}$, sendo que nos três municípios, as áreas de ILP estavam com 10, 12 e 25 anos neste sistema. Também houve aplicação de calcário em intervalos irregulares que variaram de uma a duas aplicações, com aproximadamente $2 \mathrm{t} \mathrm{ha}^{-1} \mathrm{e}$ uso de fungicidas, herbicidas e inseticidas de acordo com as recomendações técnicas.

As áreas de plantio direto foram conduzidas por sistema de rotação de culturas e manutenção permanente da cobertura do solo. As três áreas apresentavam plantio de soja no verão. Já no inverno, em Lages havia plantio de azevém e aveia e em Campo Belo do Sul foi cultivado trigo ou ficava em pousio. Em Otacílio Costa, além da soja foi plantado milho (Zea mays) no verão e nos períodos de inverno ficou em pousio. Estando as áreas com 7, 11 e 10 anos nesse sistema, respectivamente. Em todas as áreas de PD foi realizada a adubação e calagem conforme a recomendação da Comissão de Química e Fertilidade do Solo (CQFS RS/SC) (2004) para as culturas introduzidas no sistema e houve aplicação de agroquímicos. Outras informações sobre características e histórico das áreas podem ser obtidas em Rosa et al. (2015).

\section{Amostragem de besouros}

As amostras dos organismos e do solo foram coletadas, em duas épocas, inverno (julho de 2011) e verão (janeiro de 2012), de forma sistemática, em grade amostral de $3 \times 3$ pontos, espaçamento entre cada ponto de $30 \mathrm{~m} \mathrm{e}$ $20 \mathrm{~m}$ de bordadura. A amostragem resultou em 270 pontos amostrados, sendo três municípios, com cinco SUS e nove pontos em cada, em dois períodos (inverno e verão).

Para a avaliação da fauna edáfica, utilizaram-se dois métodos de amostragem, o Tropical Soil Biology and Fertility (TSBF) (ANDERSON; INGRAM, 1993) que consiste na coleta de monólitos de solo de $25 \times 25 \mathrm{~cm}$ de lado e $20 \mathrm{~cm}$ de profundidade (com a serapilheira), com auxílio de um marcador constituído de chapas de ferro galvanizadas. As amostras retiradas foram acondicionadas em sacos plásticos. No laboratório, ocorreu triagem 
manual, com o auxílio de iluminação artificial. O outro método utilizado foi de armadilhas de solo do tipo "Pitfall traps", constituídas por recipientes cilíndricos de $8 \mathrm{~cm}$ de diâmetro, com capacidade volumétrica de $500 \mathrm{~mL}$, contendo $200 \mathrm{~mL}$ de solução detergente a $0,5 \%(\mathrm{v} / \mathrm{v})$ e enterrados no solo com sua extremidade vazada nivelada com a superfície, mantidos por três dias no campo (BARETTA et al., 2014).

Em relação as análises da fauna edáfica, as amostras coletadas por armadilhas e TSBF foram triadas e todos os organismos da ordem Coleoptera foram separados e fixados em álcool $80 \%$ para conservação. Posteriormente, os coleópteros foram separados em morfo-espécies e finalmente identificados até família com auxílio de chaves dicotômicas e/ou caracterizações contidas na literatura taxonômica (LIMA, 1952-55; TRIPLEHORN; JOHNSON, 2011). O material foi depositado no laboratório de Ecologia do solo da UDESCCAV em Lages, SC.

\section{Amostragem e análises químicas e físicas em laboratório}

Para as análises químicas do solo, foram coletadas 15 subamostras ao redor de cada ponto da grade amostral na camada de $0-20 \mathrm{~cm}$, para formar uma amostra composta representativa para avaliação dos atributos (Tabela 2), conforme metodologia de Tedesco et al. (1995).

As amostras para as análises físicas (Tabela 3) foram retiradas com cilindros de aço de $5 \mathrm{~cm}$ de altura e 5 $\mathrm{cm}$ de diâmetro (indeformadas), os atributos das análises foram: densidade do solo (Ds), porosidade total (PT), microporosidade (Micro), macroporosidade (Macro) e bioporos (Bio) seguindo manual da EMBRAPA (1997). A resistência à penetração $(\mathrm{RP})$ foi avaliada por meio de um penetrômetro de bancada Marconi ${ }^{\circledR}$. Além disso, foram separadas amostras de solo com torrões para avaliação da estabilidade de agregados, diâmetro médio ponderado de agregados (DMP) e diâmetro médio geométrico de agregados (DMG) (KEMPER; CHEPIL, 1965). A granulometria do solo (areia, argila e silte) foi determinada pelo método da pipeta (GEE; BAUDER, 1986). 
Tabela 2 - Valores médios \pm desvio padrão dos atributos químicos do solo na camada de 0-20 $\mathrm{cm}$, em sistemas de Floresta nativa (FN), Reflorestamento de eucalipto (RE), Pastagem perene (PA), Integração lavoura-pecuária (ILP) e Plantio direto (PD) no Planalto Sul Catarinense.

\begin{tabular}{|c|c|c|c|c|c|}
\hline \multirow{2}{*}{$\begin{array}{l}\text { Atributo } \\
\text { químico }\end{array}$} & \multicolumn{5}{|c|}{ Sistemas de uso do solo } \\
\hline & FN & RE & PA & ILP & PD \\
\hline $\mathrm{pH} \mathrm{H}_{2} \mathrm{O}$ & $4,4 \pm 0,7$ & $4,6 \pm 0,6$ & $4,7 \pm 0,3$ & $5,3 \pm 0,1$ & $5,6 \pm 0,1$ \\
\hline pH SMP & $4,9 \pm 0,8$ & $5,1 \pm 0,9$ & $5 \pm 0,7$ & $5,8 \pm 0,3$ & $5,9 \pm 0,2$ \\
\hline $\mathbf{P}\left(\mathrm{mg} \mathrm{dm}^{-3}\right)$ & $4,3 \pm 1,0$ & $3,9 \pm 0,8$ & $3,6 \pm 1,2$ & $8,7 \pm 4,1$ & $5,8 \pm 1,5$ \\
\hline $\mathbf{K}\left(\mathrm{mg} \mathrm{dm}^{-3}\right)$ & $112 \pm 25$ & $84 \pm 22$ & $190 \pm 79$ & $135 \pm 47$ & $128 \pm 62$ \\
\hline $\mathbf{M O}\left(\mathrm{dag} \mathrm{kg}^{-1}\right)$ & $6,2 \pm 0,2$ & $4,4 \pm 0,3$ & $5,3 \pm 0,4$ & $4,7 \pm 0,7$ & $4,5 \pm 0,7$ \\
\hline $\mathbf{A l}\left(\mathrm{cmol}_{\mathrm{c}} \mathrm{dm}^{-3}\right)$ & $3,9 \pm 3,6$ & $2,8 \pm 2,9$ & $3 \pm 2,3$ & $0,5 \pm 0,1$ & $0,1 \pm 0,1$ \\
\hline $\mathbf{C a}\left(\mathrm{cmol}_{\mathrm{c}} \mathrm{dm}^{-3}\right)$ & $5,2 \pm 4,1$ & $2 \pm 1,5$ & $2,1 \pm 0,9$ & $7,2 \pm 0,4$ & $7,5 \pm 1,5$ \\
\hline $\mathbf{M g}\left(\mathrm{cmol}_{\mathrm{c}} \mathrm{dm}^{-3}\right)$ & $1,7 \pm 1,3$ & $1,8 \pm 1,5$ & $1,4 \pm 0,5$ & $3,8 \pm 1,7$ & $3,8 \pm 0,8$ \\
\hline $\mathbf{H}+\mathbf{A L}\left(\mathrm{cmol}_{\mathrm{c}} \mathrm{dm}^{-3}\right)$ & $20,8 \pm 19,3$ & $17 \pm 17,3$ & $17,5 \pm 13,6$ & $6,2 \pm 2,3$ & $4,9 \pm 1,4$ \\
\hline CTC $\left(\mathrm{cmol}_{\mathrm{c}} \mathrm{dm}^{-3}\right)$ & $27,9 \pm 14,0$ & $21 \pm 14,5$ & $21,5 \pm 12,3$ & $17,5 \pm 3,8$ & $16,5 \pm 3,6$ \\
\hline Sat $\mathbf{A l}(\%)$ & $41,7 \pm 40,9$ & $42,0 \pm 41,3$ & $40,7 \pm 25,4$ & $4,3 \pm 1,5$ & $1,4 \pm 1,5$ \\
\hline Bases $(\%)$ & $34,9 \pm 28,5$ & $30,5 \pm 24,9$ & $25,4 \pm 17,1$ & $65,1 \pm 6,3$ & $70,5 \pm 1,9$ \\
\hline K CTC $\left(\right.$ CTC $\left.^{1}\right)$ & $1,2 \pm 0,6$ & $1,2 \pm 0,4$ & $2,7 \pm 1,4$ & $2,1 \pm 1,1$ & $2,2 \pm 1,3$ \\
\hline Са CTC $\left(\mathrm{CTC}^{1}\right)$ & $25,5 \pm 21,2$ & $15,1 \pm 12,5$ & $13,8 \pm 11,1$ & $42,1 \pm 6,7$ & $45,2 \pm 1,1$ \\
\hline $\operatorname{Mg}$ CTC $\left(\mathrm{CTC}^{1}\right)$ & $8,2 \pm 7,6$ & $14,2 \pm 12,0$ & $8,9 \pm 5,7$ & $20,9 \pm 6,5$ & $23,1 \pm 2,3$ \\
\hline $\mathrm{Ca} / \mathrm{Mg}$ & $2,8 \pm 1,8$ & $1,3 \pm 0,4$ & $1,5 \pm 0,4$ & $2,3 \pm 1,2$ & $2,0 \pm 0,2$ \\
\hline $\mathrm{Ca} / \mathrm{K}$ & $17,8 \pm 13,8$ & $11,0 \pm 8,6$ & $5,7 \pm 5,1$ & $24,4 \pm 9,5$ & $33,6 \pm 28,2$ \\
\hline $\mathrm{Mg} / \mathrm{K}$ & $6,1 \pm 5,0$ & $10,2 \pm 8,4$ & $3,5 \pm 2,2$ & $13,9 \pm 9,4$ & $17,1 \pm 14,3$ \\
\hline
\end{tabular}

Médias das repetições $(\mathrm{n}=27)$.

pH: potencial hidrogeniônico; P: fósforo; K: potássio; MO: matéria orgânica; Al: alumínio; Ca: cálcio; Mg: magnésio; H+AL: acidez potencial; CTC: capacidade de trocar cátions a pH 7,0; Sat Al: saturação por alumínio; Bases: soma de bases trocáveis; $\mathrm{Ca} / \mathrm{Mg}$ : relação cálcio e magnésio; $\mathrm{Ca} / \mathrm{K}$ : relação cálcio e potássio; $\mathrm{Mg} / \mathrm{K}$ : relação magnésio e potássio.

$1 \%$ de saturação na CTC a pH 7,0. 
Tabela 3 - Valores médios \pm desvio padrão dos atributos físicos do solo em sistemas de Floresta nativa (FN), Reflorestamento de eucalipto (RE), Pastagem (PA), Integração lavoura-pecuária (ILP) e Plantio direto (PD), no Planalto Sul Catarinense.

\begin{tabular}{cccccc}
\hline Atributo & \multicolumn{5}{c}{ Sistemas de uso do solo } \\
\cline { 2 - 6 } físico & FN & RE & PA & ILP & PD \\
\hline Ds $\left(\mathrm{g} \mathrm{cm}^{-3}\right)$ & $0,89 \pm 0,02$ & $0,98 \pm 0,04$ & $0,95 \pm 0,03$ & $1,01 \pm 0,05$ & $1,01 \pm 0,10$ \\
PT $\left(\mathrm{m}^{3} \mathrm{~m}^{-3}\right)$ & $0,65 \pm 0,004$ & $0,65 \pm 0,005$ & $0,66 \pm 0,002$ & $0,65 \pm 0,005$ & $0,65 \pm 0,004$ \\
Micro $\left(\mathrm{m}^{3} \mathrm{~m}^{-3}\right)$ & $0,56 \pm 0,03$ & $0,50 \pm 0,06$ & $0,49 \pm 0,08$ & $0,47 \pm 0,02$ & $0,45 \pm 0,02$ \\
Macro $\left(\mathrm{m}^{3} \mathrm{~m}^{-3}\right)$ & $0,09 \pm 0,3$ & $0,15 \pm 0,06$ & $0,17 \pm 0,08$ & $0,19 \pm 0,02$ & $0,20 \pm 0,02$ \\
Bio $\left(\mathrm{m}^{3} \mathrm{~m}^{-3}\right)$ & $0,03 \pm 0,01$ & $0,02 \pm 0,01$ & $0,02 \pm 0,001$ & $0,01 \pm 0,004$ & $0,01 \pm 0,007$ \\
RP $\left(\mathrm{MPa}^{-3}\right)$ & $1,09 \pm 0,14$ & $1,53 \pm 0,24$ & $1,84 \pm 0,29$ & $1,94 \pm 0,12$ & $1,85 \pm 0,35$ \\
Areia $(\mathrm{dag} \mathrm{kg-1})$ & $42,9 \pm 14,0$ & $35,5 \pm 22,5$ & $34,3 \pm 16,4$ & $25,9 \pm 21,2$ & $19,1 \pm 11,0$ \\
Argila $(\mathrm{dag} \mathrm{kg})$ & $37,7 \pm 10,1$ & $46,2 \pm 21,0$ & $46,5 \pm 18,2$ & $49,9 \pm 24,9$ & $47,1 \pm 20,8$ \\
Silte $(\mathrm{dag} \mathrm{kg})$ & $19,3 \pm 5,3$ & $18,3 \pm 1,6$ & $19,2 \pm 5,0$ & $24,1 \pm 5,9$ & $33,8 \pm 10,1$ \\
DMP $(\mathrm{mm})$ & $5,55 \pm 0,36$ & $5,58 \pm 0,40$ & $5,68 \pm 0,35$ & $5,51 \pm 0,08$ & $5,62 \pm 0,14$ \\
DMG $(\mathrm{mm})$ & $7,69 \pm 0,65$ & $7,79 \pm 0,56$ & $7,87 \pm 0,60$ & $7,59 \pm 0,07$ & $7,71 \pm 0,24$ \\
\hline Médias das repetições $(\mathrm{n}=27)$. & & & &
\end{tabular}

Ds: densidade do solo; PT: porosidade total; Micro: microporosidade; Macro: macroporosidade; Bio: bioporos; RP: resistência à penetração; DMP: diâmetro médio ponderado de agregados; DMG: diâmetro médio geométrico de agregados.

\section{Análise dos dados}

As avaliações foram realizadas ao nível de sistema de uso do solo, usando o valor de três municípios (nove amostras por município) como réplica verdadeira $(\mathrm{n}=3 \times$ $9=27$ ) em cada SUS e nas duas épocas (inverno e verão) analisadas separadamente. Os dados obtidos a partir dos dois métodos de amostragem da fauna (armadilhas e TSBF) também foram analisados separados, ou seja, os resultados são apresentados por SUS, época do ano e tipo de coleta. Os índices de Pielou (J) e diversidade de Shannon-Wiener $\left(\mathrm{H}^{\prime}\right)$ foram calculados pela biblioteca Vegan presente no programa estatístico R (R DEVELOPMENT CORE TEAM, 2011).

Os dados de abundância foram submetidos à análise estatística multivariada. Inicialmente a uma Detrended Correspondence Analysis (DCA), a fim de obter o comprimento do gradiente da matriz de dados. Considerando que este comprimento foi inferior a três $(<$ 3), ou seja, com resposta linear, optou-se por fazer Análise de Componentes Principais (ACP), para cada uma das épocas estudadas (inverno e verão) já que foi detectado efeito de época $(\mathrm{p} \leq 0,05)$.

A abundância de famílias foi utilizada como variável resposta e os atributos químicos e físicos do solo como variáveis ambientais explicativas da distribuição dos coleópteros pelos diferentes SUS nas ACPs. As variáveis explicativas colineares foram identificadas através do
Variance Inflation Factor (VIF) e por operações de forward selection, usando sucessivas Redundancy Analysis (RDAs) com base em permutações por teste de Monte-Carlo, retirando as que apresentaram colinearidade e selecionando as que melhor explicaram a variação dos dados $(p \leq 0,05)$. Somente as variáveis significativas das RDAs foram posteriormente utilizadas na ACP como variáveis ambientais explicativas passivas para as mudanças observadas das famílias de Coleoptera nos SUS. Para todas as análises estatísticas multivariadas utilizou-se o software estatístico CANOCO versão 4.5 (ter BRAAK; SMILAUER, 2002).

\section{RESULTADOS E DISCUSSÃO}

\section{Riqueza e diversidade de famílias de Coleoptera}

Foi coletado um total de 1.437 coleópteros, distribuídos em 32 famílias. Do total registrado, 26 famílias foram coletadas em armadilhas de queda e 24 por TSBF, sendo 18 famílias compartilhadas por ambos os métodos de coleta, oito ocorreram apenas em armadilhas de solo e seis exclusivamente pelo método TSBF (Tabela 4).

Favero, Souza e Oliveira. (2011), estudando área de "Cordilheiras" no Mato Grosso do Sul (MS), identificaram dez famílias em seu estudo de áreas em regeneração utilizando armadilhas de queda. Portilho et al. (2011) amostraram seis famílias em diferentes sistemas de uso do solo, pelo método TSBF, no estado do MS. 
Segundo estes mesmos autores, as famílias mais abundantes identificadas em seus trabalhos foram
Scarabaeidae e Staphylinidae, mesmo resultado encontrado no presente estudo (Tabela 4).

Tabela 4 - Famílias de Coleoptera amostradas e número de indivíduos em sistemas de Floresta nativa (FN), Reflorestamento de eucalipto (RE), Pastagem perene (PA), Integração lavoura-pecuária (ILP) e Plantio direto (PD), no inverno (I) e verão (V) no Planalto Sul Catarinense.

\begin{tabular}{|c|c|c|c|c|c|c|c|c|c|c|c|c|c|c|c|c|c|c|c|c|}
\hline \multirow[b]{2}{*}{ Família } & \multicolumn{20}{|c|}{ Sistemas de Uso do Solo } \\
\hline & $\mathrm{FN}$ & $\begin{array}{c}\mathrm{RE} \\
\mathrm{Ar}\end{array}$ & $\begin{array}{l}\text { PA } \\
\text { adi }\end{array}$ & $\begin{array}{l}\text { ILP } \\
\text { as (I }\end{array}$ & PD & $\mathrm{FN}$ & $\begin{array}{r}\mathrm{RE} \\
\mathrm{A}_{1}\end{array}$ & $\begin{array}{l}\text { PA } \\
\text { nadi }\end{array}$ & $\begin{array}{l}\text { ILP } \\
\text { has }\end{array}$ & PD & $\mathrm{FN}$ & & $\begin{array}{c}\text { PA } \\
\text { TSB }\end{array}$ & $\begin{array}{l}\text { ILP } \\
\text { (I) }\end{array}$ & PD & $\mathrm{FN}$ & & $\begin{array}{l}\text { PA } \\
\text { SBF }\end{array}$ & $\begin{array}{l}\text { ILP } \\
\text { V) }\end{array}$ & PD \\
\hline Staphylinidae & 4 & 7 & 3 & 1 & - & 215 & 25 & 26 & 4 & 30 & 81 & 18 & 34 & 39 & 24 & 50 & 16 & 17 & 45 & 21 \\
\hline Carabidae & - & 2 & - & 18 & 3 & 20 & 6 & 2 & 82 & 22 & 9 & 5 & 6 & 34 & 6 & - & 2 & 4 & 41 & 42 \\
\hline Scarabaeidae & 22 & 1 & 1 & - & 6 & 81 & 5 & 5 & 26 & 2 & 5 & 1 & 6 & 5 & 3 & 2 & 3 & 7 & 1 & 3 \\
\hline Leiodidae & 3 & 3 & - & - & - & 20 & 3 & - & 7 & 15 & 2 & - & - & - & - & 2 & - & - & - & 2 \\
\hline Chrysomelidae & 2 & - & 2 & 2 & 1 & 1 & 1 & 3 & 4 & 1 & 2 & 6 & 4 & 6 & 2 & 7 & 2 & 2 & 2 & 2 \\
\hline Ptiliidae & 2 & - & 4 & 1 & 2 & 7 & 6 & 3 & - & 2 & - & - & - & - & - & - & - & 1 & - & - \\
\hline Tenebrionidae & - & - & - & - & - & 5 & 1 & - & - & 2 & - & 1 & - & 1 & 6 & - & 2 & - & 3 & - \\
\hline Scydmaenidae & - & - & 1 & 1 & 1 & 2 & - & - & 1 & - & 2 & 5 & 1 & 1 & - & 2 & 1 & - & - & 1 \\
\hline Silvanidae & - & - & 1 & - & - & - & 1 & - & 1 & 5 & - & - & - & - & 5 & - & - & - & - & 5 \\
\hline Ptilodactylidae & - & - & - & - & - & 3 & 1 & - & - & - & - & - & - & - & - & 7 & - & - & 1 & 1 \\
\hline Pselaphydae & - & - & - & - & - & - & - & - & - & - & 5 & - & - & - & - & 5 & 1 & 1 & - & - \\
\hline Lathridiidae & - & - & - & - & 3 & - & - & - & 2 & 5 & - & - & - & 1 & - & - & - & - & - & - \\
\hline Anobiidae & - & - & - & - & - & - & - & - & - & - & 3 & - & - & - & - & - & - & 4 & - & - \\
\hline Anthicidae & - & - & - & 1 & - & - & - & 2 & - & 1 & - & - & - & 1 & - & - & - & - & - & 1 \\
\hline Curculionidae & - & - & - & - & 1 & - & - & - & - & - & - & 1 & 1 & - & - & - & 3 & - & - & - \\
\hline Elateridae & - & - & - & - & - & 1 & 1 & 1 & - & - & 1 & - & - & - & - & 1 & - & - & - & - \\
\hline Phalacridae & - & - & - & - & - & - & - & - & - & - & 1 & 1 & - & - & - & - & 2 & - & 1 & - \\
\hline Chelonariidae & - & - & - & - & - & 1 & - & - & - & - & - & 1 & - & - & - & 2 & - & - & - & - \\
\hline Histeridae & - & - & - & - & - & - & - & 4 & - & - & - & - & - & - & - & - & - & - & - & - \\
\hline Salpingidae & - & - & 2 & 1 & - & - & - & - & - & - & - & - & - & - & - & - & - & - & - & 1 \\
\hline Dryopidae & - & - & - & - & - & - & - & - & - & - & 2 & - & - & - & - & - & - & - & - & - \\
\hline Hydrophididae & - & - & - & - & - & 2 & - & - & - & - & - & - & - & - & - & - & - & - & - & - \\
\hline Lagriidae & - & - & - & - & 1 & - & - & - & - & - & - & - & - & 1 & - & - & - & - & - & - \\
\hline Scaphidiidae & - & - & - & - & - & - & 1 & - & - & - & - & 1 & - & - & - & - & - & - & - & - \\
\hline Brentidae & - & - & - & - & - & 1 & - & - & - & - & - & - & - & - & - & - & - & - & - & - \\
\hline Cerambycidae & - & - & - & - & - & - & 1 & - & - & - & - & - & - & - & - & - & - & - & - & - \\
\hline Cicindelidae & - & - & - & - & - & - & - & - & - & - & - & - & - & - & - & - & - & - & 1 & - \\
\hline Corylophidae & - & - & - & - & - & 1 & - & - & - & - & - & - & - & - & - & - & - & - & - & - \\
\hline Eucnemidae & - & - & - & - & - & - & - & - & - & - & - & - & - & - & - & 1 & - & - & - & - \\
\hline Malachiidae & - & - & - & - & - & 1 & - & - & - & - & - & - & - & - & - & - & - & - & - & - \\
\hline Nitidulidae & - & - & - & - & - & - & - & - & - & 1 & - & - & - & - & - & - & - & - & - & - \\
\hline Tetratomidae & - & - & - & 1 & - & - & - & - & - & - & - & - & - & - & - & - & - & - & - & - \\
\hline Total & 33 & 13 & 14 & 26 & 18 & 361 & 52 & 46 & 127 & 86 & 113 & 40 & 52 & 89 & 46 & 79 & 32 & 36 & 95 & 79 \\
\hline
\end{tabular}

No inverno, pelo método das armadilhas de solo, a família Scarabaeidae apresentou maior abundância de indivíduos, principalmente na FN. Já no verão foi a família Staphylinidae que obteve maior abundância e em ambas as épocas a família Carabidae foi mais representativa no sistema ILP. Com o método TSBF, a família Staphylinidae foi melhor representada nas duas épocas. Quando observadas as abundâncias das famílias nas duas 
amostragens e épocas de coleta, percebe-se que juntamente com Staphylinidae e Scarabaeidae, a família Carabidae foi bastante representativa, principalmente nos sistemas PD e ILP (Tabela 4).

Essas famílias foram as que mais contribuíram para os maiores valores de abundâncias totais de coleópteros nos sistemas FN e ILP. Algumas espécies das famílias Staphylinidae e Carabidae são importantes agentes de controle biológico e relacionados com ambientes mais complexos e naturais, como florestas nativas e sensíveis a alteração microclimática, pois a temperatura e a umidade do solo são fatores que regulam sua distribuição (GARLET et al., 2015). Essas condições favoráveis para a sobrevivência desses indivíduos podem ter sido encontradas também nos ambientes de ILP.

Quanto à família Scarabaeidae, um grande número desses coleópteros pode estar associado a maior disponibilidade de recursos florísticos e resíduos vegetais e animais na $\mathrm{FN}$, fatores que estão muito relacionados à presença marcante desse grupo, que possui espécies com variados hábitos alimentares (FAVERO; SOUZA; OLIVEIRA, 2011). Portanto, a participação destas famílias pode indicar condições mais estáveis de habitat nos SUS.

Os maiores valores de diversidade de ShannonWiener ( $\left.\mathrm{H}^{\prime}\right)$ e equabilidade de Pielou $(J)$ das famílias de coleópteros foram observados em RE, no método de amostragem TSBF, quando avaliado separadamente (Tabela 5). Apenas no período do verão para os coleópteros amostrados por armadilhas de solo os maiores índices foram obtidos em PD e no inverno em PA, seguidos por RE. Esses resultados demonstram que $O$ sistema FN não obteve maior diversidade de famílias frente aos demais SUS como se esperava e, provavelmente, a intervenção antrópica e entrada de animais de produção sem controle de gestão nos fragmentos florestais, podem trazer perdas para a diversidade de famílias de coleópteros.

Tabela 5 - Valores dos índices de diversidade de ShannonWiener ( $\left.H^{\prime}\right)$ e de Pielou (J) para famílias de Coleoptera, em sistemas de Floresta nativa (FN), Reflorestamento de eucalipto (RE), Pastagem perene (PA), Integração lavourapecuária (ILP) e Plantio direto (PD) no Planalto Sul Catarinense.

\begin{tabular}{cccccc}
\hline & FN & RE & PA & ILP & PD \\
\cline { 2 - 5 } & \multicolumn{5}{c}{ Armadilhas inverno } \\
\hline $\mathrm{H}^{\prime}$ & 1,08 & 1,16 & 1,81 & 1,15 & 1,08 \\
$\mathrm{~J}$ & 0,47 & 0,50 & 0,79 & 0,50 & 0,47 \\
\hline \multicolumn{5}{c}{ Armadilhas verão } \\
\hline $\mathrm{H}^{\prime}$ & 1,28 & 1,74 & 1,49 & 1,13 & 1,77 \\
$\mathrm{~J}$ & 0,46 & 0,63 & 0,54 & 0,41 & 0,64 \\
\hline
\end{tabular}

\begin{tabular}{cccccc}
\hline \multicolumn{5}{c}{ TSBF inverno } \\
\cline { 1 - 5 } $\mathrm{H}^{\prime}$ & 1,18 & 1,68 & 1,12 & 1,29 & 1,43 \\
$\mathrm{~J}$ & 0,45 & 0,64 & 0,43 & 0,49 & 0,54 \\
\hline \multicolumn{5}{c}{ TSBF verão } \\
\hline $\mathrm{H}^{\prime}$ & 1,36 & 1,70 & 1,52 & 1,10 & 1,38 \\
$\mathrm{~J}$ & 0,50 & 0,63 & 0,56 & 0,41 & 0,51 \\
\hline
\end{tabular}

Em estudo realizado por Favero, Souza e Oliveira (2011), os autores encontraram valores de 1,96 e 1,72 para H', com coleópteros amostrados por armadilhas. Portilho et al. (2011) associaram as melhores condições de diversidade de invertebrados do solo a sistemas com rotação de culturas como ILP e PD, quando amostrados por TSBF, em MS, indicando que sistemas conservacionistas favorecem o equilíbrio dinâmico do solo.

\section{Análise de componentes principais (ACP) com variáveis ambientais explicativas para a comunidade de Coleoptera}

As ACPs, para os coleópteros amostrados por armadilha, tanto no inverno quanto no verão (Figura 1), demonstraram distinção entre os sistemas de uso do solo, através da relação entre a componente principal 1 (CP1) e a componente principal 2 (CP2).

Para as famílias amostradas por armadilhas, no inverno (Figura 1a), o eixo da CP1 explicou 26,0\% da variabilidade dos dados e o eixo da CP2 explicou $17,6 \%$, somados, os eixos explicaram $43,6 \%$ da variabilidade total. As famílias Leiodidae e Staphylinidae relacionaram-se positivamente com os sistemas $\mathrm{RE}$ e $\mathrm{FN}$ e os atributos MO e Bio contribuiram para explicar essa variação. Já as famílias Salpingidae, Carabidae e Scydmaenidae tiveram maior relação com ILP e PA, Lathridiidae, Scarabaeidae e Ptiliidae com o PD.

Muitos coleópteros da família Staphylinidae vivem na matéria orgânica vegetal ou animal e sua forte relação com a matéria orgânica já foi evidenciada em outros trabalhos (MUELLER et al., 2016), além de ser muito sensível as alterações microclimáticas. Esses fatores podem ter contribuído para a maior relação desta família e também Leiodidae com as áreas florestais, que possuem uma menor intensificação de uso do solo e geralmente favorecem um microclima mais estável, pois as coberturas florestais diminuem a radiação solar abaixo do dossel, mantém a temperatura e a umidade relativa do ar próximo a superfície e há manutenção dos resíduos vegetais no solo.

A família Ptiliidae, compreende os menores besouros conhecidos, com espécies medindo menos de 1 $\mathrm{mm}$ de comprimento, vivem onde há matéria orgânica em 
decomposição e geralmente se alimentam de esporos de fungos que encontram em material vegetal em decomposição, formigueiros, etc. É uma família muito abundante e se distribui amplamente por todo o mundo (MAJKA; SÖRRENSON, 2010). Sua ocorrência pode estar ligada à variável Ds e também ao estado de agregação do solo, representado pela variável DMP (Figura 1a).

A maior Ds nos sistemas PD e PA (Figura 1a) pode ter sido causada pelo pisoteio animal ou por trânsito de máquinas. Essa modificação estrutural pode provocar alterações no fluxo de água e nutrientes e, consequentemente, afetar a atividade biológica. Entretanto, quando observados os valores da Ds na Tabela 3, percebese que mesmo sendo mais elevada em PD não é um valor crítico e não afetou de forma negativa a família Ptiilidae. Além disso, pode haver compactação natural se o agricultor não conseguir manter a qualidade do sistema PD ao longo do tempo, mas se a gestão é realizada de forma adequada o acúmulo de matéria orgânica e aumento da biodiversidade edáfica nesse sistema tende a reduzir a compactação.
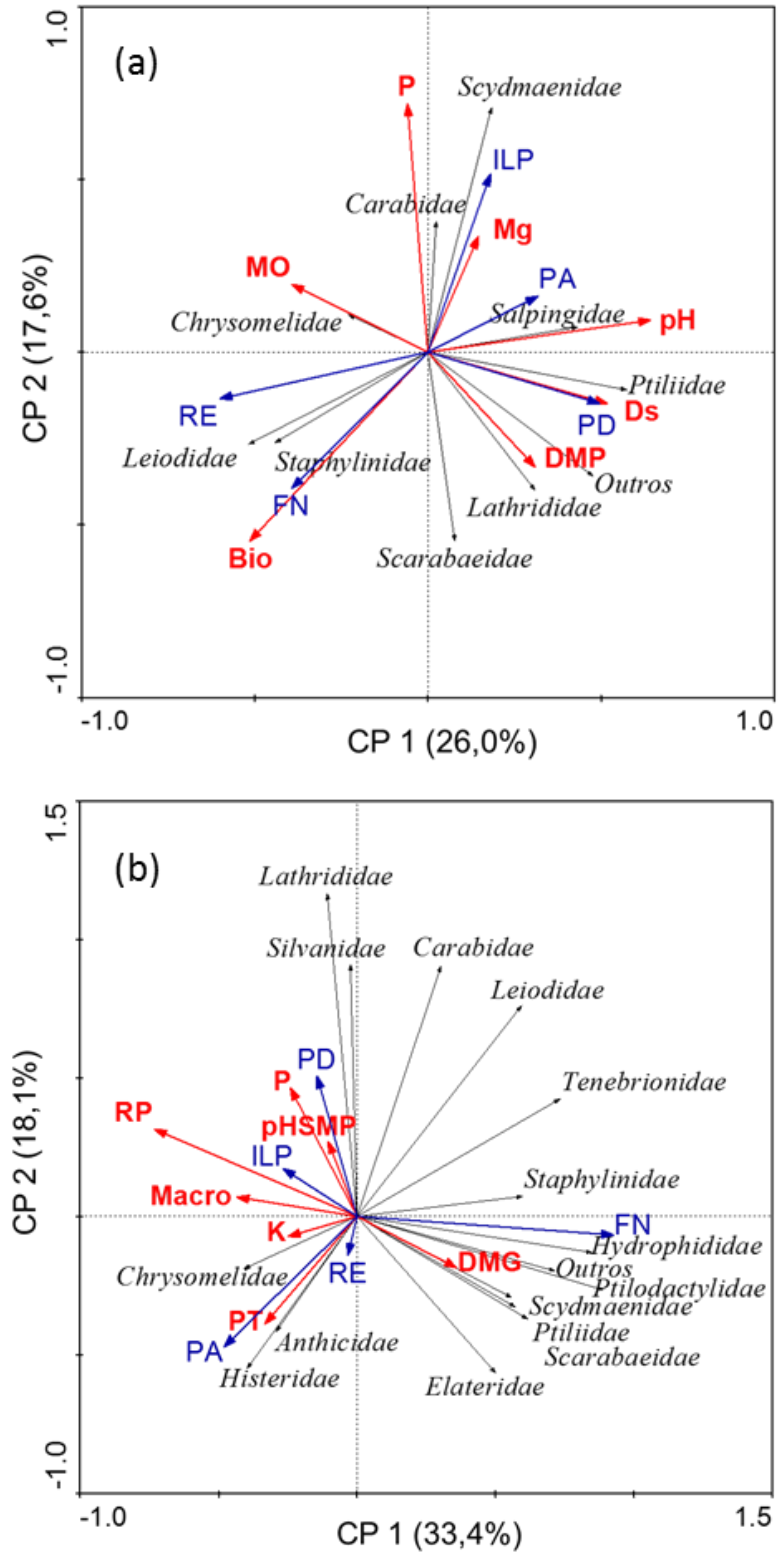

Figura 1 - Análise de componentes principais para as famílias de Coleoptera (preto) amostradas por armadilhas, em relação aos sistemas de uso do solo (azul) e as variáveis ambientais (vermelho) utilizadas como explicativas, no inverno (a) e verão (b), no Planalto Sul Catarinense.

Floresta nativa (FN); Reflorestamento de eucalipto (RE); Pastagem perene (PA); Integração lavoura-pecuária (ILP); Plantio direto (PD); Matéria orgânica (MO); Bioporos (Bio); Diâmetro médio ponderado de agregados (DMP); Densidade do solo (Ds); Pontencial hidrogeniônico $(\mathrm{pH})$; Resistência a penetração (RP); Macroporos (Macro); Porosidade total (PT); Diâmetro média geométrico de agregados (DMG); Famílias raras com apenas um indivíduo (Outros).

Por outro lado, a maior relação com o aumento do DMP pode indicar que as modificações no ambiente 
estão contribuindo para a formação de agregados, ao promover a aproximação das partículas minerais. No entanto, solos compactados podem apresentar elevada estabilidade de agregados em água e, consequentemente, acarretar em maiores valores de DMP (CONTE et al., 2011).

$\mathrm{Na}$ ACP para as famílias amostradas por armadilhas de solo no verão (Figura 1b), o eixo da CP1 explicou $33,4 \%$ da variabilidade dos dados e o eixo da CP2 apenas $18,1 \%$. Nesta figura, a maior parte das famílias está relacionada com o sistema $\mathrm{FN}$ com maior valor da variável DMG. O sistema PA apresentou maior associação com as famílias Chrysomelidae, Histeridae e Anthicidae, e os valores mais elevados da variável PT contribuíram para explicar essa condição. Já o sistema PD apresentou maior abundância de coleópteros das famílias Lathridiidae, Silvanidade e Carabidae, favorecidas pelos maiores valores de $\mathrm{P}, \mathrm{pH}$ SMP.

O sistema RE por outro lado, obteve menor participação na ordenação. A variável DMG ajudou na explicação do elevado número de famílias em $\mathrm{FN}$, dando destaque para as famílias Scarabaeidae, Scydmaenidae e Pitiliidae (Figura 1b).

No verão a $\mathrm{FN}$ apresentou a maior diversidade de famílias e estas, com variados hábitos alimentares, desde fitófagas, coprófagas, predadoras, saprófagas, entre outros, que ocupam nichos tróficos diferenciados e interagem com o meio onde vivem de diferentes formas. Por exemplo, algumas espécies de besouros da família Scarabaeidae promovem a fragmentação e transporte do material orgânico e sua movimentação para diferentes locais pode contribuir para melhorar a aeração do solo e o estado de agregação, características que podem estar associadas a FN e ao DMG (Figura 1b).

Os coleópteros da família Histeridae, por sua vez, são predadores tanto na fase larval quanto na fase adulta e utilizam diferentes ambientes para se alimentarem. Assim, recursos em decomposição atraem os detritívoros que compõem parcela importante da alimentação desse grupo. Portanto, são atraídos por fezes de animais em busca de larvas de outros invertebrados. Sua presença em áreas de PA (Figura 1b) pode ser condicionada por atuarem como predadores. A associação desse grupo com PT pode ser relacionada ao seu papel como agentes de manejo físico, pois alguns coleópteros dessa família frequentam galerias perfuradas no solo em busca de alimento (LOPES et al., 2006).

De acordo com a ACP para as famílias amostradas por TSBF, no inverno (Figura 2a), o eixo da CP1 explicou $21,4 \%$ da variabilidade dos dados e o eixo da CP2 explicou $18 \%$. Os sistemas RE e FN tiveram relação com maior número de famílias, sendo Curculionidae e Chrysomelidae mais associadas ao RE e os maiores valores de areia no solo. Já as famílias Staphylinidae e Phalacridae ficaram relacionadas com a $\mathrm{FN}$, com melhores condições das variáveis $\mathrm{MO}$ e Bio. Além destas, a relação $\mathrm{Ca} / \mathrm{Mg}$ contribuiu para explicar a participação das famílias Leiodidae e Dryopidae em FN. Os sistemas PA e PD ficaram posicionados no canto inferior esquerdo da ordenação, juntamente com as maiores abundâncias de coleópteros das famílias Scarabaeidae, Silvanidae, Tenebrionidae e Pselaphydae. Já ILP ficou mais associado as famílias Carabidae e Anobiidae, juntamente aos maiores valores da variável $P$.

No verão (Figura 2b), com a amostragem por TSBF, a CP1 explicou 22,5\% da variabilidade dos dados e a CP2 explicou 16\%. Nesse período o sistema FN ficou separado dos outros sistemas e apresentou relação com maior número de famílias, especialmente Staphylinidae, Chrysomelidae e Leiodidae, explicada pelos maiores valores dos atributos MO e PT. Carabidae ficou mais relacionada ao sistema PD e ILP devido aos valores mais elevados das variáveis $\mathrm{P}$ e $\mathrm{pH}$. No sistema RE, houve associação mais fraca com Tenebrionidae e PA com alguns grupos como Scarabaeidae e Anobiidae.

Os carabídeos (Carabidae) são sensíveis as mudanças antrópicas e podem ser considerados indicadores do impacto de cultivos, sendo negativamente afetados pela agricultura intensiva, controle mecanizado de ervas daninhas e pelo fogo. Entretanto, alguns grupos dessa família são mais generalistas e toleram perturbações ambientais e outros aumentam sua ocorrência com a adubação do solo (WINK et al., 2005). Essas condições do solo nos sistemas PD e ILP, avaliados no presente estudo, podem ter favorecido alguns grupos específicos de Carabidae, principalmente pelos maiores teores de $\mathrm{P}$, como observado na Tabela 2 e nas Figuras 1 e 2. Martins et al. (2012), verificaram que áreas de plantio direto e fragmento florestal tem maior diversidade de Carabidae e Staphylinidae, quando comparada com área de plantio convencional, demonstrando que a composição da vegetação é fundamental para a distribuição destes coleópteros do solo.

Observando todos os gráficos de ACP, relacionados às famílias, em ambos métodos de amostragem (Aramadilhas de solo e TSBF) e nas duas estações (inverno e verão), destacou-se a associação da família Staphylinidae com FN e o atributo MO, indicando a elevada frequência dessa família em solos com maior aporte de resíduos orgânicos. Esse comportamento é devido à sua abundância e ampla distribuição, demonstrando assim um provável potencial para ser considerada como família bioindicadora das propriedades dos solos.

De modo geral, as maiores abundâncias dos coleópteros distribuídos na $\mathrm{FN}$ demonstraram que a 
cobertura vegetal e a disponibilidade de recursos são atrativos a esses invertebrados, que se beneficiam com as melhores condições para alimentação e abrigo. Além disso, os atributos químicos e físicos do solo influenciam no estabelecimento dos grupos nos diferentes sistemas de uso do solo e algumas famílias de coleópteros podem ser favorecidas pelo uso e manejo adotado nas áreas de produção, pois alteram as propriedades do solo.
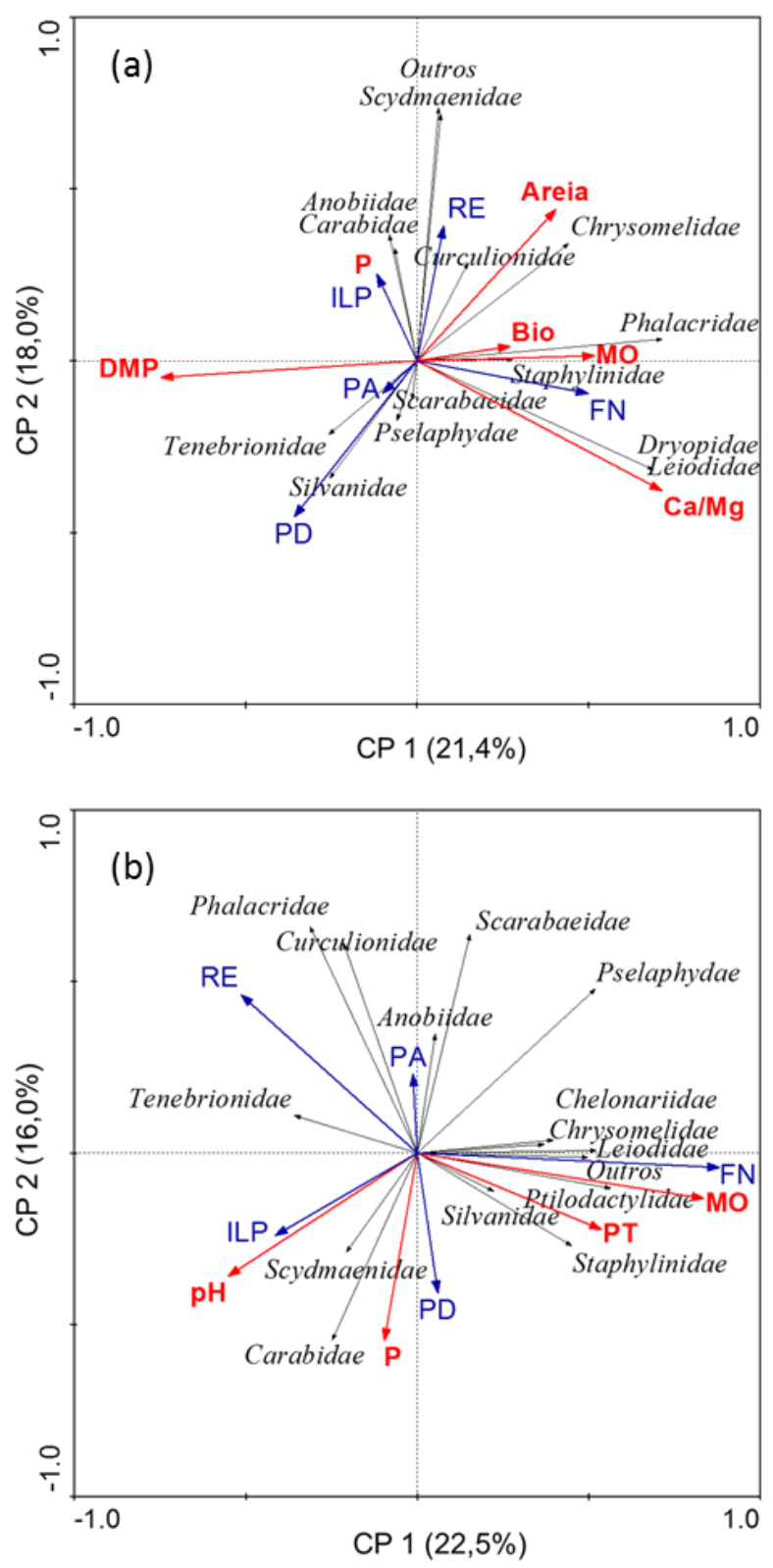

Figura 2 - Análise de componentes principais para as famílias de Coleoptera (preto) amostradas por TSBF em relação aos sistemas de uso do solo (azul) e as variáveis ambientais (vermelho) utilizadas como explicativas, no inverno (a) e verão (b), no Planalto Sul Catarinense.

Floresta nativa (FN); Reflorestamento de eucalipto (RE); Pastagem perene (PA); Integração lavoura-pecuária (ILP); Plantio direto (PD); Diâmetro médio ponderado de agregados (DMP); Bioporos (Bio); Matéria orgânica (MO); Porosidade total (PT); Potencial hidrogeniônico $(\mathrm{pH})$; Famílias raras com apenas um indivíduo (Outros).

\section{CONCLUSÕES}

A diversidade de famílias de Coleoptera é influenciada pela época de amostragem (inverno e verão) e por alguns atributos químicos e físicos do solo, os quais explicam a diversidade e abundância de famílias distribuídas entre a Floresta Nativa (FN), Reflorestamento de Eucalipto (RE), Pastagem Perene (PA), Integração Lavoura-Pecuária (ILP) e Plantio Direto (PD) no Planalto Sul Catarinense.

O sistema de uso do solo com maior abundância de famílias de coleópteros foi $\mathrm{FN}$, especialmente por apresentar maiores valores de $\mathrm{MO}$, bioporos e agregação do solo. Dentre as famílias, a Staphylinidae apresentou maior associação com $\mathrm{FN}$, além de ter sido a família com mais representantes, independente do SUS. Outra família que se destacou foi Carabidae, fortemente relacionada aos sistemas PD e ILP devido aos teores mais elevados de P.

Sugere-se que sejam realizados novos estudos que verifiquem o potencial das famílias de Coleoptera como indicadoras das condições do ambiente, com o intuito de reforçar esses dados e de gerar mais informações em outros sistemas de uso do solo.

\section{AGRADECIMENTOS}

Os autores agradecem a FAPESC (Processo no 6309-6/2011) e ao Conselho Nacional de Desenvolvimento Científico e Tecnológico (CNPq Processo: 307162/2015-0) pelo apoio financeiro. P.N. Pompeo agradece ao Programa de Monitoria de Pósgraduação (PROMOP/UDESC) pela concessão de bolsa de Mestrado. D. Baretta agradece ao CNPq pela Bolsa de Produtividade Científica (Processo no 307162/2015-0). 


\section{REFERÊNCIAS}

ALMEIDA, S. S. P.; LOUZADA, J. N. C. Community structure of Scarabaeinae (Scarabaeidae: Coleoptera) in Brazilian savannah phytophysiognomies and its importance for conservation. Neotropical Entomology. v. 38, n. 1, p. 3243, 2009.

ANDERSON, J. M.; INGRAM, J. S. I. Tropical soil biology and fertility: a handbook of methods. 2. ed. Wallingford: CAB International, 1993. 171p.

ASHFORD O. S.; FOSTER, W. A.; TURNER, B. L.; SAYER, E. J.; SUTCLIFFE, L.; TANNER, E. V. J. Litter manipulation and the soil arthropod community in a lowland tropical rainforest. Soil Biology and Biochemistry. v. 62, p. 5-12, 2013.

BARETTA D.; MAFRA, Á. L.; SANTOS, J. C. P.; AMARANTE, C. V. T. DO; BERTOL, I. Análise multivariada da fauna edáfica em diferentes sistemas de preparo e cultivo do solo. Pesquisa Agropecuária Brasileira. v. 41, p. 1675-1679, 2006.

BARETTA, D.; BARTZ, M. L. C.; FACHINI, I.; ANSELMI, R.; ZORTÉA, T.; BARETTA, C. D. R. M. Soil fauna and its relation with environmental variables in soil management systems. Revista Ciência Agronômica. v. 45, n. 5, p. 871-879, 2014.

CQFS - COMISSÃO DE QUÍMICA E FERTILIDADE DO SOLO, RS/SC. Manual de adubação e de calagem para os estados do Rio Grande do Sul e Santa Catarina. $10^{\mathrm{a}}$ ed. Porto Alegre: SBCS/NRS, 2004. 400p.

CONTE O.; FLORES, J. P. C.; CASSOL, L. C.; ANGHINONI, I.; CARVALHO, P. C. de F.; LEVIEN, R.; WESP, C. de L. Evolução de atributos físicos de solo em sistema de integração lavoura-pecuária. Pesquisa Agropecuária Brasileira. v. 46, n. 10, p. 1301-1309, 2011.

EMBRAPA - EMPRESA BRASILEIRA DE PESQUISA AGROPECUÁRIA. Centro Nacional de Pesquisa de Solos. Manual de métodos de análises de solo. $2^{\mathrm{a}}$. ed. Rio de Janeiro: Embrapa; 1997.

FARIAS, P. M. de; ARELLANO, L.; HERNÁNDEZ, M. I. M.; ORTIZ, S. L. Response of the copro-necrophagous beetle (Coleoptera: Scarabaeinae) assemblage to a range of soil characteristics and livestock management in a tropical landscape. Journal of Insect Conservation. v. 19, p. 947-960, 2015.
FAVERO, S.; SOUZA, H. A.; OLIVEIRA, A. K. M. Coleoptera (Insecta) as forest fragmentation indicators in the Rio Negro sub-region of the Pantanal, Mato Grosso do Sul, Brazil. Brazilian Journal of Biology. v. 71, n. 1, p. 291-295, 2011.

GARLET J.; COSTA, E. C.; BOSCARDIN, J.; MACHADO, D. N.; PEDRON, L. Fauna de Coleoptera edáfica em eucalipto sob diferentes sistemas de controle químico da matocompetição. Floresta e Ambiente. v. 22, p. 239-248, 2015.

GEE, G. W.; BAUDER, J. W. Particle-size analysis. In: KLUTE, A. editor. Methods of soil analysis. Madison: American Society of Agronomy. p.383-411, 1986.

KEMPER, W. D.; CHEPIL, W. S. Size distribution of aggregation. In: BLACK, C. A., editor. Methods of soil analysis. Madison: American Society of Agronomy. p.499510, 1965.

KROMP, B. Carabid beetles in sustainable agriculture: A review on pest control efficacy, cultivation impacts and enhancement. Agriculture, Ecosystems and Environment. v. 74, p. 187-228, 1999.

LEE, M. S.; ALBAJES, R. Monitoring carabid indicators could reveal environmental impacts of genetically modified maize. Agricultural and Forest Entomology. v. 18, p. 238-249, 2016.

LIIRI M.; HÄSÄ, M.; HAIMI, J.; SETÄLÄ, H. History of land use intensity can modify the relationship between functional complexity of the soil fauna and soil ecosystem services - A microcosm study. Applied Soil Ecology. v. 55, p. 53-61, 2012.

LIMA, A. C. Insetos do Brasil. Coleópteros $1^{\mathrm{a}}, 2^{\mathrm{a}}$ e $3^{\mathrm{a}}$ parte. Rio de Janeiro: Escola Nacional de Agronomia; 1952-55. 372,323 e 288 p.

LOPES, W. D. Z.; LOPES, W. C. Z.; COSTA, F. H. da; BALIEIRO, J. C. C. DE; PRADO, Â. P. do. Abundância e sazonalidade de histerídeos (Coleoptera) associados ao esterco de granja aviária da Região Nordeste do Estado de São Paulo, Brasil. Revista Brasileira de Entomologia. v. 50, n. 4, p. 492-497, 2006.

MAJKA, C. G.; SÖRENSSON, M. Featherwing beetles (Coleoptera: Ptiliidae) of the Atlantic Maritime Ecozone. In: MCALPINE, D. F.; SMITH, I. M., editores. Assessment 
of species diversity in the Atlantic Maritime Ecozone. Ottawa: NRC Research Press, 2010. p. 433-438.

MARTINS, I. C. F.; CIVIDANES, F. J.; IDE, S.; HADDAD, G. Q. Diversity and habitat preferences of Carabidae and Staphylinidae (Coleoptera) in two agroecosystems. Bragantia. v. 71, p. 471-480, 2012.

MUELLER, K. E.; EISENHAUER, N.; REICH, P. B.; HOBBIE, S. E.; CHADWICK, O. A.; CHOROVER, J.; DOBIES, T.; HALE, C. M.; JAGODZIŃSKI, A. M.; KALUCKA, I.; KASPROWICZ, M.; KIELISZEWSKAROKICKA, B.; MODRZYŃSKI, J.; ROŻEN, A.; SKORUPSKI, M.; SOBCZYK, L.; STASIŃSKA, M.; TROCHA, L. K.; WEINER, J.; WIERZBICKA, A.; OLEKSYN, J. Light, earthworms, and soil resources as predictors of diversity of 10 soil invertebrate groups across monocultures of 14 tree species. Soil Biology and Biochemistry. v. 92, p. 184-198, 2016.

PORTILHO, I. I. R.; CREPALDI, R. A.; BORGES, C. D.; SILVA, R. F. da; SALTON, J. C.; MERCANTE, F. M. Fauna invertebrada e atributos físicos e químicos do solo em sistemas de integração lavoura-pecuária. Pesquisa Agropecuária Brasileira. v. 46, n. 10, p. 1310-1320, 2011.

R DEVELOPMENT Core Team. R: a language and environment for statistical computing. [2.12.2]. Vienna: R Foundation for Statistical Computing, 2011.

ROSA, M. G.; KLAUBERG FILHO, O.; BARTZ, M. L. C.; MAFRA, Á. L.; SOUSA, J. P. F. A. de; BARETTA, D. Macrofauna edáfica e atributos físicos e químicos em sistemas de uso do solo no Planalto Catarinense. Revista Brasileira de Ciência do Solo. v. 39, p.1544-1553, 2015.

TEDESCO, M. J.; GIANELlO, C.; BISSANI, C. A.; BOHNEN, H.; VOLKWEISS, S. J. Análise de solo, plantas e outros materiais. $2^{\mathrm{a}}$. ed. Porto Alegre: Universidade Federal do Rio Grande do Sul, 1995. 174p.

ter BRAAK, C. J. F.; SMILAUER, P. CANOCO reference manual and CanoDraw for Windows user's guide: software for canonical community ordination (version 4.5). Ithaca: Microcomputer Power, 2002. 500p.

TRIPLEHORN, C. A.; JOHNSON, N. F. Estudo dos insetos. São Paulo: Cengage Learning, 2011. 816p.

WINK, C.; GUEDES, J. V. C.; FAGUNDES, C. K.; ROVEDDER, A. P. Insetos edáficos como indicadores da qualidade ambiental. Revista de Ciências Agroveterinárias. v. 4, n. 1, p. 60-71, 2005. 